\title{
Imagenología coronaria: ultrasonido intravascular (IVUS)
}

\author{
Sergio H. Vásquez ${ }^{a}$, Boris E. Vesga ${ }^{a, b, *}$ y Héctor J. Hernández ${ }^{a}$ \\ a Instituto del Corazón de Bucaramanga S.A, Bucaramanga, Colombia \\ ${ }^{\mathrm{b}}$ Departamento de Medicina Interna - Universidad Industrial de Santander, Bucaramanga, Colombia
}

Recibido el 4 de abril de 2017; aceptado el 10 de noviembre de 2017

Disponible en Internet el 3 de enero de 2018

\section{PALABRAS CLAVE \\ Enfermedad \\ coronaria; \\ Aterosclerosis; \\ Ultrasonido \\ intravascular; \\ Placa aterosclerótica}

\section{KEYWORDS}

Coronary disease;

Atherosclerosis;

Intravascular

ultrasound;

Atherosclerotic

plaque
Resumen La enfermedad aterosclerótica compromete toda la longitud del vaso arterial coronario, en diferentes grados de severidad. La arteriografía coronaria ha sido el estándar de oro en la evaluación imagenológica de las arterias coronarias. Estudios histopatológicos han demostrado que subestima la severidad de la aterosclerosis aún en los sitios de mayor compromiso. La complejidad de la enfermedad coronaria hace necesario implementar nuevas herramientas en la evaluación anatómica y funcional de la aterosclerosis. El ultrasonido intravascular (IVUS) ofrece imágenes con mejor resolución espacial ya que permite evaluar la severidad de las lesiones y aporta información respecto a composición de la placa, disposición dentro de la pared vascular y evaluación del fenómeno de reestenosis y trombosis de los stent coronarios.

(c) 2017 Publicado por Elsevier España, S.L.U. en nombre de Sociedad Colombiana de Cardiología y Cirugía Cardiovascular. Este es un artículo Open Access bajo la licencia CC BY-NC-ND (http:// creativecommons.org/licenses/by-nc-nd/4.0/).

\section{Coronary imaging: intravascular ultrasound (IVUS)}

Abstract Atherosclerotic disease involves the entire length of the coronary artery, to varying degrees of severity. Coronary arteriography has been the gold standard in imaging assessments of the coronary arteries. Histopathological studies have shown that it underestimates the severity of atherosclerosis even in areas with the greatest involvement. The complexity of coronary disease requires the implementation of new tools for the anatomical and functional assessment of atherosclerosis. Intravascular ultrasound (IVUS) offers images with better spatial resolution, since it allows the evaluation of the severity of the lesions and provides information regarding plaque composition, location within the vascular wall, and evaluation of the coronary stent restenosis and thrombosis phenomenon.

(c) 2017 Published by Elsevier España, S.L.U. on behalf of Sociedad Colombiana de Cardiología y Cirugía Cardiovascular. This is an open access article under the CC BY-NC-ND license (http: / / creativecommons.org/licenses/by-nc-nd/4.0/).

\footnotetext{
* Autor para correspondencia.
}

Correos electrónicos: borisvesga@institutodelcorazon.com, o.boris@uis.edu.co (B.E. Vesga). 


\section{Objetivo}

Desarrollar un consenso de aproximación en cuanto al diagnóstico intravascular coronario mediante la técnica de ultrasonido en sujetos adultos con enfermedad coronaria, con el fin de unificar criterios diagnósticos y de manejo en los servicios de Cardiología intervencionista de Colombia, con miras a cumplir metas de calidad y reunir lo recomendado por la literatura actual.

\section{Alcance}

Los servicios de Hemodinamia y Cardiología intervencionista son parte de las instituciones de salud públicas y privadas con alto nivel de complejidad. En estos se realizan procedimientos diagnósticos invasivos en sujetos sintomáticos de enfermedad coronaria, y se ofrece el respectivo manejo intervencionista como parte integral del tratamiento de la enfermedad coronaria. Por consiguiente, se requiere que los procedimientos estén estandarizados con el fin de aplicarlos con parámetros de calidad en Colombia.

\section{Definiciones}

\section{Ultrasonido intravascular}

Técnica imagenológica de la pared arterial en la que se utilizan transductores que convierten ondas de ultrasonido en imágenes bi o tridimensionales en forma de cortes transversales o longitudinales de un segmento de arteria.

\section{Responsables}

Profesionales de Salud Médicos con formación en Hemodinamia y Cardiología intervencionista debidamente certificados para ejecutar su ejercicio en el territorio colombiano.

\section{Desarrollo}

\section{Población objeto}

Pacientes con edad igual o mayor a 18 años, con enfermedad arterial coronaria conocida por angiografía coronaria y que requieran imágenes intracoronarias.

\section{Metodología}

Se llevó a cabo una revisión bibliográfica teniendo en cuenta las guías presentadas por European Heart Society - European APCI, American Heart Association, American College of Cardiology, Society SCAI, Sociedad Latinoamericana de Cardiología Intervencionista, y portales como TCTmd y PCR online, de donde se toman elementos para adaptarlos al entorno colombiano.

\section{Introducción}

Desde hace más de 50 años, la angiografía coronaria con contraste es el estándar de oro para el diagnóstico de enfermedad coronaria. Sin embargo, pese a que es adecuada para el diagnóstico de estenosis de la luz arterial, tiene como limitación el no poder definir las características de la pared vascular, así como la significancia de lesiones intermedias y el impacto funcional de las mismas. Como solución a la definición de la caracterización de la pared se han desarrollado herramientas imagenológicas como el ultrasonido intravascular (IVUS) y la tomografía de coherencia óptica (OCT), las cuales ofrecen imágenes con mejor resolución espacial y permiten hacer una evaluación más objetiva de la severidad de las lesiones además de aportar información respecto a la composición de la placa y la disposición dentro de la pared vascular, al igual que ha mostrado utilidad en el estudio del fenómeno de la reestenosis y la trombosis de los stent.

\section{Generalidades}

La enfermedad aterosclerótica compromete, en diferentes grados de severidad, la longitud del vaso epicárdico coronario. Desde el punto de vista angiográfico, la severidad de la enfermedad se establece al comparar el segmento "enfermo o de calibre más reducido" con el segmento adyacente de calibre "normal o sin lesión'. Aquellos segmentos puntuales en los que la enfermedad genera una reducción intraluminal mayor del $70 \%$ del diámetro, se reconocen como lesiones estenosantes angiográficamente severas. Sin embargo, la complejidad de la enfermedad coronaria ha enseñado la importancia de implementar otras herramientas en la evaluación anatómica y funcional de las lesiones ${ }^{1}$.

Los estudios histopatológicos han demostrado que la angiografía con contraste subestima la severidad de la enfermedad aún en los sitios más afectados ${ }^{2}$. Se reconoce, dentro del crecimiento y desarrollo de la placa de ateroma, el fenómeno de remodelación positiva de la pared vascular en el que el vaso, mediante un desplazamiento externo de la pared (aumento del área de la membrana elástica externa EEM), se "adapta" a la placa en crecimiento, generando un aumento del área de la placa, con el objetivo de conservar el área de lumen vascular. Cuando este mecanismo llega a su límite por la progresión de la placa de ateroma, no solo aumenta el área de la EEM sino que empieza a reducir el área luminal, siendo evidente en la angiografía ${ }^{3}$.

\section{Principios del ultrasonido intravascular}

A través de un catéter guía 5 o 6 French $(\mathrm{Fr})$ de diámetro y sobre una guía de angioplastia 0,014 pulgadas se avanza el catéter de ultrasonido, de calibre $3 \mathrm{Fr}$ y que en su punta aloja el transductor de ultrasonido. Bajo el mismo principio utilizado en las ecografías, el transductor emite y recibe ondas de ultrasonido que posteriormente son procesadas en escala de grises, reconstruyendo una imagen circunferencial de la sección de vaso a analizar. El transductor puede estar compuesto por un único cristal piezoeléctrico, que rota sobre su propio eje a una frecuencia de $45 \mathrm{MHZ}$ (sistema mecánico) o por múltiples cristales de pequeño tamaño y con disposición circunferencial, que se activan de forma secuencial a una frecuencia de $20 \mathrm{MHZ}$ (sistema electrónico). El procedimiento al igual que la angioplastia coronaria, se realiza bajo efecto de heparina no fraccionada en dosis de 
anticoagulación, en tanto que la adquisición de imágenes se hace posterior a la administración de nitroglicerina intracoronaria para minimizar el vasoespasmo coronario y así subvalorar el área del vaso.

\section{Características de la imagen}

La imagen de ultrasonido permite evaluar las capas que conforman la pared vascular. La íntima se ve reflejada como una capa fina ecodensa (brillante), difícil de visualizar en pacientes jóvenes. La media es la capa adyacente y tiene aspecto ecolúcido (oscuro) debido a la menor presencia de colágeno. La adventicia es la capa más externa, tiene aspecto ecodenso y representa el límite externo del vaso. El engrosamiento de la pared con la separación del borde de la íntima con la media, es el hallazgo principal de la enfermedad aterosclerótica y se conoce como placa de ateroma (fig. 1).

La ecodensidad de la placa de ateroma está en relación con el contenido histopatológico de la misma y también se representa en escala de grises. El parámetro de comparación es el aspecto de la adventicia. Una placa de ateroma puede tener aspecto ecodenso (similar a la adventicia; brillante, cercano al color blanco) o ecolúcido (oscuro, cercano al color negro); por ejemplo, las placas lipídicas tienen aspecto ecolúcido, mientras que las placas ricas en colágeno y calcio tienen aspecto ecodenso. Teniendo en cuenta que la composición de la placa de ateroma es heterogénea y dada la limitación de la escala de grises en la diferenciación de esta composición, el software cuenta con una herramienta conocida como histología virtual, la cual permite una mejor caracterización del contenido de la placa.

Mediante la utilización de software, se puede delinear cada capa y calcular diámetros y áreas. La delimitación de la íntima revela el área luminal, mientras que la delimitación de la media y adventicia (lámina elástica externa, EEM) revela el área del vaso y la diferencia entre estas dos áreas revela el área de ateroma contenido en la media. Cuando se adquieren las imágenes mediante el dispositivo de tracción automático (pullback), al conocer la velocidad de captura de imágenes $(0,5 \circ 1 \mathrm{~mm} / \mathrm{s})$, se puede determinar la longitud de la lesión en estudio; con estas medidas se puede calcular el volumen de placa aterosclerótica (carga de placa), valoración que se ha utilizado en investigación clínica para evaluar fenómenos de modificación de la misma como son la estabilización, regresión o progresión de la placa.

\section{Aplicaciones diagnósticas del ultrasonido intravascular}

El IVUS ayuda a caracterizar lesiones localizadas a nivel ostial, en tronco coronario izquierdo, en segmentos tortuosos, bifurcaciones y lesiones excéntricas o severamente calcificadas.

Las lesiones que reducen angiográficamente el diámetro del vaso entre 40 y $70 \%$ son un reto diagnóstico para definir si requieren intervencionismo. Al compararse el IVUS contra la angiografía coronaria cuantitativa y la estimación visual, habrá mejor precisión y reproducibilidad al evaluar diámetros, áreas del vaso y volúmenes de placa.

El IVUS, al estimar el área luminal mínima (medición del área luminal mínima), permite establecer el grado de severidad de una lesión; los valores por debajo del punto de corte para tronco coronario o vaso coronario, se han correlacionado con pruebas fisiológicas como la medición del flujo fraccional de reserva (FFR).

En un estudio de comparación de IVUS y FFR, en el que usaron puntos de corte de 0,75 , se encontró que la medición del área luminal mínima menor a $3 \mathrm{~mm}^{2}$ tenía una sensibilidad del $83 \%$ y una especificidad del $92,3 \%$, mientras que en el IVUS las áreas de estenosis mayor al 60\% tenían sensibilidad del $92 \%$ y especificidad del $88,5 \%$; al utilizar ambos criterios se logra sensibilidad y especificidad del $100 \%{ }^{4}$.

En otro estudio, al utilizar el FFR con valor de corte 0,8 , se documentó que una medición del área luminal mínima $\geq 4 \mathrm{~mm}^{2}$ confiere un valor predictivo negativo mayor al $94 \%$, en tanto que una medición del área luminal mínima de $3,09 \mathrm{~mm}^{2}$ establece para un FFR de 0,8 , el punto de equilibrio entre sensibilidad $(69,2 \%)$ y especificidad $(79,5 \%)^{5}$.

Finalmente, los valores de corte de medición del área luminal mínima que se correlacionan con FFR menor de 0,8 (fisiológicamente significativo) varían con base en el calibre del vaso: medición del área luminal mínima $\leq 2,4 \mathrm{~mm}^{2}$ para
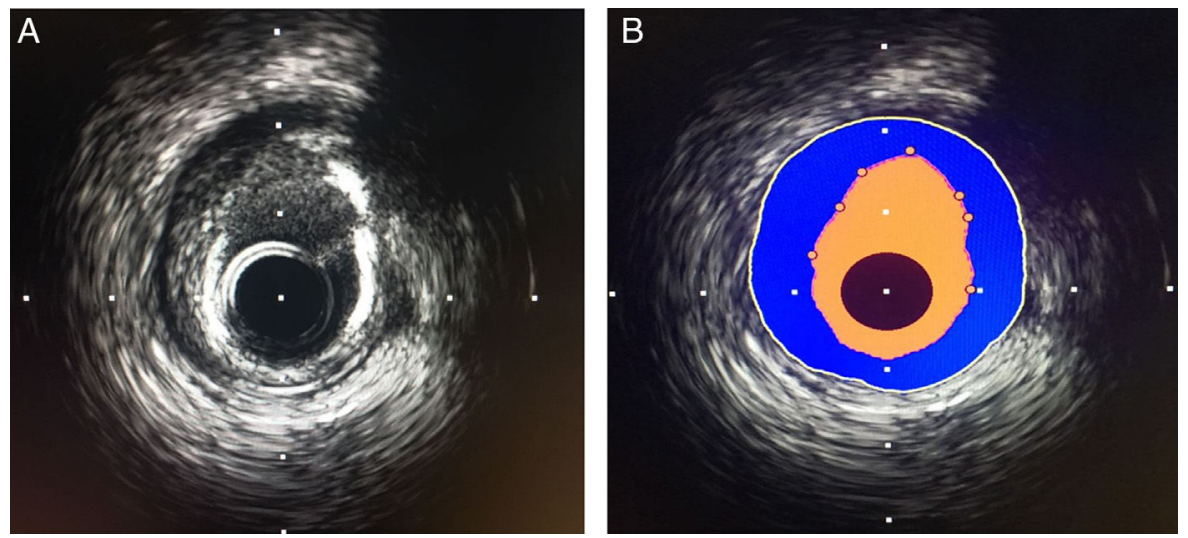

Figura 1 A. Representación imagenológica de la pared vascular mediante IVUS, B. Representación esquemática de la pared vascular; área luminal color amarillo, intima (puntos amarillos), media representada por línea blanca, área azul corresponde a área de ateroma. 
vasos de pequeño calibre (diámetro 2,5-3,0 mm), medición del área luminal mínima $\leq 2,7 \mathrm{~mm}^{2}$ para vasos de mediano calibre (diámetro $3,0-3,5 \mathrm{~mm}$ ) y medición del área luminal mínima $\leq 3,6 \mathrm{~mm}^{2}$ para vasos de gran calibre (diámetro mayor de $3,5 \mathrm{~mm})^{5}$. Para la evaluación de lesiones en el tronco coronario izquierdo se emplea como corte una medición del área luminal mínima $\leq 5,9 \mathrm{~mm}^{2}$, con base en sensibilidad del $93 \%$ y especificidad del $95 \%$.

\section{Vasculopatía del injerto cardiaco}

Esta entidad de etiología desconocida, catalogada como predictor de mortalidad e infarto de miocardio en pacientes trasplantados, afecta las arterias coronarias epicárdicas del injerto que, desde el punto de vista histopatológico, presenta engrosamiento de la íntima mayor a $0,5 \mathrm{~mm}^{7}$. Al ser un compromiso difuso, puede ser pasado por alto con la angiografía, en cuyo caso el IVUS ofrece mejor sensibilidad y especificidad en su diagnóstico cuando se evalúa en el postrasplante a las cuatro a seis semanas y al primer año ${ }^{8}$. El IVUS, además, es útil para evaluar la respuesta al tratamiento de esta patología con pravastatina ${ }^{9}$ y everolimus ${ }^{10}$.

\section{Estimación del tamaño real del vaso}

En escenarios como la angioplastia coronaria primaria, oclusiones crónicas donde situaciones como alta carga trombótica, vasoespasmo y llenado incompleto del vaso durante la angiografía dificultan visualizar y dimensionar una arteria, la medición precisa y reproducible de las dimensiones del vaso de referencia, permite seleccionar de forma adecuada el tamaño del stent, balón de angioplastia u oliva de aterectomía, lo cual disminuye la probabilidad de complicaciones derivadas de una subestimación (retroceso elástico, reestenosis o trombosis por ganancia luminal incompleta) o por sobreestimación de las dimensiones del vaso (disección, ruptura).

\section{Ayuda durante la angioplastia con stent}

Desde su implementación el IVUS ha ayudado en el refinamiento de la técnica para el implante de los stent. A pesar de una angiografía de aspecto óptimo, los primeros reportes de ultrasonido revelaban una expansión insuficiente y una aposición incompleta de los strut. El IVUS permite documentar la aposición adecuada y completa de los strut del stent al igual que la identificación y entendimiento del mecanismo de reestenosis y trombosis. Además, permite detectar la presencia de calcio superficial denso circunferencial que lleve a la necesidad de utilizar dispositivos de aterectomía previo a la colocación del stent; la realización de IVUS lleva a un cambio en la estrategia de angioplastia en un 20 a $40 \%$ de los casos.

La medición del área luminal mínima y del diámetro mínimo de la luz intrastent (MLD) posterior al implante de un stent e indexado a las dimensiones del vaso de referencia, ha ayudado a definir el concepto de expansión adecuada del mismo, siendo necesario en algunos casos posdilataciones con balones de mayor tamaño o no complacientes. Hoy en día es claro que el valor de medición del área luminal mínima es un predictor de reestenosis ${ }^{11}$.

Es importante tener en cuenta que pese a que el IVUS ayuda a mejorar los resultados postangioplastia con mayor medición del área luminal mínima resultante y mejor aposición de los strut, su uso rutinario no ha demostrado disminución significativa en desenlaces duros como mortalidad o infarto (MACE); aunque datos obtenidos en registros ${ }^{12}$ y algunos ensayos clínicos pequeños ${ }^{13-15}$ sugieren que se presentan menores tasas de reestenosis, trombosis y reintervención de las lesiones tratadas (TLR). Estudios como el ADAPT-DES ${ }^{13}$ estiman que su uso puede reducir la trombosis de stent y MACE.

Igual situación se da entre la asociación de trombosis de stent medicados y malaposición de los strut; un estudio encontró que los pacientes con trombosis tardía presentaban malaposición en el $77 \%$ de los casos, versus $12 \%$ en el grupo de control ${ }^{14}$. Sin embargo, otros estudios no han encontrado dicha asociación ${ }^{15}$.

\section{Estudio del mecanismo de la reestenosis intrastent}

La reestenosis intrastent se presenta como respuesta cicatricial al daño focal en la pared vascular generada por la angioplastia. Se caracteriza por el crecimiento exagerado de la neoíntima, generando patrones de localización focal, difusa 0 en alguno de los bordes del stent. Se estima que hasta el $20 \%$ de las reestenosis son consecuencia de subexpansión del stent (medición del área luminal mínima pos implantación de stent menor del $70 \%$ del área luminal de referencia) ${ }^{16}$.

La localización de la RIS es importante para definir la conducta a tomar; cuando es focal y entre los bordes del stent se trata con angioplastia con balón medicado a alta presión; cuando es difusa o se extiende más allá de alguno de los bordes del stent se trata mediante el implante de un nuevo stent medicado ${ }^{17,18}$. Se recomienda revisar el documento de manejo de la reestenosis intrastent que hace parte de esta guía.

\section{Identificación de complicaciones: disección coronaria}

La disección coronaria es la causa más frecuente de cierre abrupto del vaso durante el intervencionismo coronario. El rápido reconocimiento y manejo de esta complicación tiene impacto en la morbimortalidad. El IVUS ha demostrado, en comparación con la angiografía, ser una herramienta con mayor sensibilidad para documentar y caracterizar esta complicación. Existe una clasificación que organiza los tipos de disección según la profundidad de la misma (intimal, media, adventicia), al igual que la presencia de hematoma de pared $^{19}$.

\section{Seguridad del procedimiento}

La complicación más frecuente durante la realización del IVUS es el espasmo coronario (hasta un $3 \%$ de los casos), que se resuelve con la administración de nitroglicerina. Complicaciones mayores como disección, trombosis, espasmo 

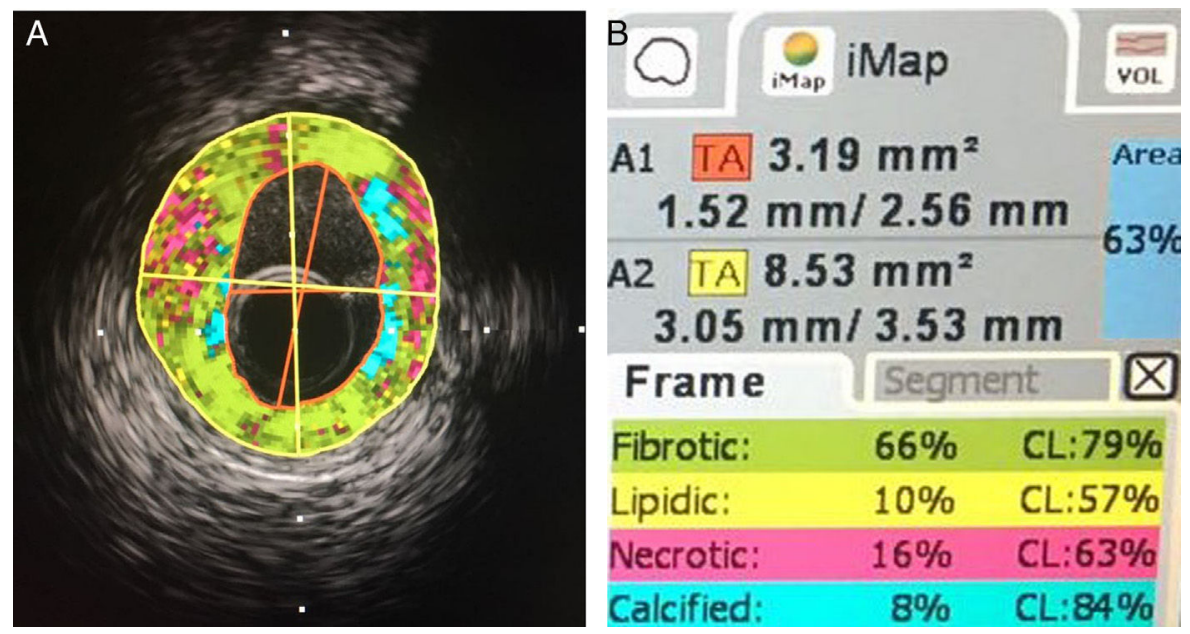

Figura 2 Histología virtual. A la izquierda, corte de arteria con evaluación de composición de placa. A la derecha: porcentaje de cada componente.

refractario e infarto ocurren en menos de $0,15 \%$ de los casos $^{22}$.

\section{Histología virtual derivada del IVUS}

El análisis de la señal de dispersión de radiofrecuencia se conoce como histología virtual; esta técnica permite mejorar la caracterización de los componentes de la placa y puede llegar a orientar sobre la estrategia de manejo durante intervencionismo cardiovascular (fig. 2).

\section{Conclusiones}

- El IVUS es una herramienta útil en el diagnóstico de enfermedad coronaria porque evalúa lesiones estenosantes angiográficamente intermedias y en la vasculopatía postrasplante.

- El IVUS es una herramienta útil antes de la intervención percutánea de lesiones, ya que determina el tamaño del vaso, las características de la placa, el mecanismo de reestenosis y la trombosis de un stent.

- El IVUS es una herramienta útil posterior a la intervención percutánea, pues optimiza el stent, evalúa la cobertura adecuada de la lesión y documenta complicaciones.

- El IVUS es una herramienta útil en investigación: respuesta terapéutica a estatinas, hipolipemiantes, antiproliferativos, conservación/pérdida de medición del área luminal mínima.

\section{Conflicto de intereses}

Ninguno.

\section{Bibliografía}

1. Vlodaver Z, Frech R, Van Tassel RA. Correlation of the antemortem coronary arteriogram and the postmortem specimen. Circulation. 1973;47:162-9.
2. Arnett EN, Isner JM, Redwood DR. Coronary artery narrowing in coronary Heart disease: comparison of cineangiographic and necropsy findings. Ann Intern Med. 1979;91:350-6.

3. Glagov S, Weisenberg E, Zarins CK. Compensatory enlargement of human atherosclerotic coronary arteries. N Engl J Med. 1987;316:1371-5.

4. Takagi A, Tsurumi Y, Ishii Y. Clinical potential of intravascular ultrasound for physiological assessment of coronary stenosis: relationship between quantitative ultrasound tomography and pressure-derived fractional flow reserve. Circulation. 1999;100:250-5.

5. Ben-Dor I, Torguson R. Deksissa. Intravascular ultrasound lumen area parameters for assessment of physiological ischemia by fractional flow reserve in intermediate coronary artery stenosis. Cardiovasc Revasc Med. 2012;13:177-82.

6. Jasti V, Ivan E, Yalamanchili V. Correlations between fractional flow reserve and intravascular ultrasound in patients with an ambiguous left main coronary artery stenosis. Circulation. 2004;110:2831-6.

7. Tuzcu EM, Kapadia SR, Sachar R. Intravascular ultrasound evidence of angiographically silent progression in coronary atherosclerosis predicts long-term morbidity and mortality after cardiac transplantation. J Am Coll Cardiol. 2005;45: $1538-42$.

8. Uretsky BF, Murali S, Reddy PS. Development of coronary artery disease in cardiac transplant patients receiving immunosuppressive therapy with cyclosporine and prednisone. Circulation. 1987;76:827-34.

9. Kobashigawa JA, Katznelson S, Laks H. Effect of pravastatin on outcomes after cardiac transplantation. N Engl J Med. 1995;333:621-7.

10. Eisen HJ, Tuzcu EM, Dorent R. Everolimus for the prevention of allograft rejection and vasculopathy in cardiac-transplant recipients. N Engl J Med. 2003;349:847-58.

11. de Feyter PJ, Kay P, Disco C. Reference chart derived from post-stent implantation intravascular ultrasound predictors of 6 month expected restenosis on quantitative coronary angiography. Circulation. 1999;100:1777-83.

12. Orford JL, Denktas AE, Williams BA. Routine intravascular ultrasound scanning guidance of coronary stenting is not associated with improved clinical outcomes. Am Heart J. 2004;148: 501-6.

13. Witzenbichler B, Maehara A, Weisz G. Use of IVUS reduces stent thrombosis: results from the prospective, multicenter ADAPTDES study. Circulation. 2014;129:463-70. 
14. Cook S, Wenaweser P, Togni M. Incomplete stent apposition and very late stent thrombosis after drug-eluting stent implantation. Circulation. 2007;115:2426-34.

15. Fujii K, Carlier SG, Mintz GS. Stent underexpansion and residual reference segment stenosis are related to stent thrombosis after sirolimus-eluting stent implantation: an intravascular ultrasound study. J Am Coll Cardiol. 2005;45:995-8.

16. Hoffmann R, Mintz GS, Dussaillant GR. Patterns and mechanisms of in-stent restenosis. A serial intravascular ultrasound study. Circulation. 1996;94:1247-54.

17. Lemos PA, Saia F, Ligthart JM. Coronary restenosis after sirolimus-eluting stent implantation: morphological description and mechanistic analysis from a consecutive series of cases. Circulation. 2003;108:257-60.

18. Castagna MT, Mintz GS, Leiboff BO. The contribution of "mechanical" problems to in-stent restenosis: an intravascular ultrasonographic analysis of 1090 consecutive in-stent restenosis lesions. Am Heart J. 2001;142:970-4.

19. Mintz GS, Nissen SE, Anderson WD. American College of Cardiology Clinical Expert Consensus Document on Standards for Acquisition, Measurement and Reporting of Intravascular Ultrasound Studies (IVUS). A report of the American College of Cardiology Task Force on Clinical Expert Consensus Documents. J Am Coll Cardiol. 2001;37:1478-92. 